\title{
Novel variants in COL4A4 and COL4A5 are rare causes of FSGS in two unrelated families
}

\author{
Stephanie L. Hines', Anjali Agarwal², Mohamedanwar Ghandour², Nabeel Aslam¹, Ahmed N. Mohammad ${ }^{2,3}$ and \\ Paldeep S. Atwal $\mathbb{B}^{2}$
}

\begin{abstract}
We report two female patients with focal segmental glomerulosclerosis and chronic kidney disease. The first patient was found to have a heterozygous, de novo, pathogenic variant in COL4A5 (c.141+1G>A, IVS2+1G>A), which is associated with Alport syndrome. The second patient was found to have a heterozygous, likely pathogenic variant in COL4A4 (c.2842G>T). Both these variants in COL4A5 and COL4A4 are novel, and they were detected using whole exome sequencing and gene panel testing, respectively. Additionally, we discuss the complexities of diagnosis in such cases and the benefits of using the abovementioned diagnostic approaches.
\end{abstract}

\section{Introduction}

Focal segmental glomerulosclerosis (FSGS) is a progressive kidney disease that can be either a primary renal disorder or secondary due to other etiologies, including genetic etiologies ${ }^{1}$. It is the culprit of approximately $40 \%$ of nephrotic syndrome cases in adults and $20 \%$ in children ${ }^{2,3}$. As the name suggests, FSGS is characterized by focal [less than $50 \%$ of glomeruli are affected in light microscopy (LM)] and segmental [less than $50 \%$ of a glomerular tuft is affected] glomerular sclerosis and by varying degrees of foot process effacement ${ }^{2}$. Pathogenic variants in genes that are specific to the function and structure of podocytes, such as TRPC6, ACTN4, WT1, CD2AP, INF2, NPHS2, and PLCE1, have been reported to cause familial FSGS $^{4}$. Pathogenic variants in collagen type IV genes (COL4A3, COL4A4, and COL4A5) are related to a spectrum of disorders with heterogeneous clinical manifestations.

Alport syndrome (AS), which is caused by glomerular membrane defects, has X-linked and autosomal dominant/recessive modes of inheritance. Classical AS has X-linked inheritance due to pathogenic variants in COL4A5 (86\% of cases) and usually presents in early childhood with microscopic or gross hematuria, which progresses to end stage renal disease ${ }^{5}$.The remaining 15\% of AS has autosomal inheritance and is thought to be due to pathogenic variants in the COL4A3 and COL4A4 genes. Thin basement membrane nephropathy (TBMN), which is also characterized by glomerular membrane abnormalities, has been linked to COL4A3 and COL4A4 as well ${ }^{6}$. Renal disorders causing glomerular basement membrane structural abnormalities are known collectively as collagen IV nephropathies (COL4Ns) ${ }^{7}$. FSGS typically presents with proteinuria, often in the nephrotic range, which progresses over months to years to a reduction in glomerular filtration rate (GFR). COL4A3 and COL4A4, which are located on chromosome 2, have been associated with $\mathrm{FSGS}^{8}$. Herein, we present two families with novel COL4A4 and COL4A5 variants diagnosed by massively parallel exome sequencing and discuss the complexities of managing these genetic results. 


\section{Materials and methods}

To identify the molecular bases of the patients' phenotypes, whole exome sequencing was utilized in the first patient, while the second proband was tested with a nephrotic syndrome/focal segmental glomerulosclerosis sequencing panel.

\section{Results}

The exome sequencing revealed a heterozygous, de novo, pathogenic variant in COL4A5 (c.141+1G $>\mathrm{A}$, IVS2 $+1 \mathrm{G}>\mathrm{A}$ ), a heterozygous, paternally inherited, pathogenic variant in $A B C A 4$ (c.2588G $>$ C, p.G863A) and a heterozygous, maternally inherited, likely pathogenic variant in $A B C A 4$ (c.203C $>$ G, p.P68R). The nephrotic syndrome/focal segmental glomerulosclerosis sequencing panel detected a heterozygous, likely pathogenic variant in COL4A4 (c.2842G>T).

\section{Family 1}

Our first proband is a 52-year-old female with stage IV chronic kidney disease (estimated GFR of $28.2 \mathrm{~mL} / \mathrm{min}$ ), microalbuminuria (microalbumin/creatinine ratio of $206 \mathrm{mg} / \mathrm{g}$ ) and microscopic hematuria. Her kidney disease first started at age 7 , when she presented with microscopic hematuria, which was thought to be benign. At that point in time, her kidney function was normal, and she subsequently did well until age 34 , when she developed proteinuria (3 grams of protein/24-h urine), hypertension and creatinine clearance of $45 \mathrm{~mL} / \mathrm{min}$. She underwent a renal biopsy, which showed FSGS with no foot process effacement, suggestive of a secondary form. She was started on $30 \mathrm{mg}$ prednisone daily, which resulted in remission of her proteinuria. A renal ultrasound scan showed multiple bilateral renal cysts (Fig. 1), though no family history of ADPKD was found. However, her son was also diagnosed with microscopic hematuria at age 7 . In addition to the kidney disease, the patient has chronic arthritis, for which she takes lowdose prednisone; hypermobile joints; migraine headaches; and macular degeneration, which was diagnosed at age 40. She underwent a brain MRI to evaluate the headaches, and the result was normal except for a mild Chiari malformation. A genetic etiology to her kidney disease was suspected, and samples from the proband's mother, father and affected son were submitted for variant segregation analysis by whole exome sequencing (WES). The patient was found to have a heterozygous, de novo, pathogenic variant in COL4A5 (c.141+1G>A, IVS2 $+1 G>A$ ), which is associated with Alport syndrome. As it is de novo, her siblings are unlikely to be at risk for the disorder, although germline mosaicism cannot be excluded. Her son (18 years of age) carries this variant, giving him the genetic diagnosis of Alport syndrome as well. Moreover, the patient was found to be

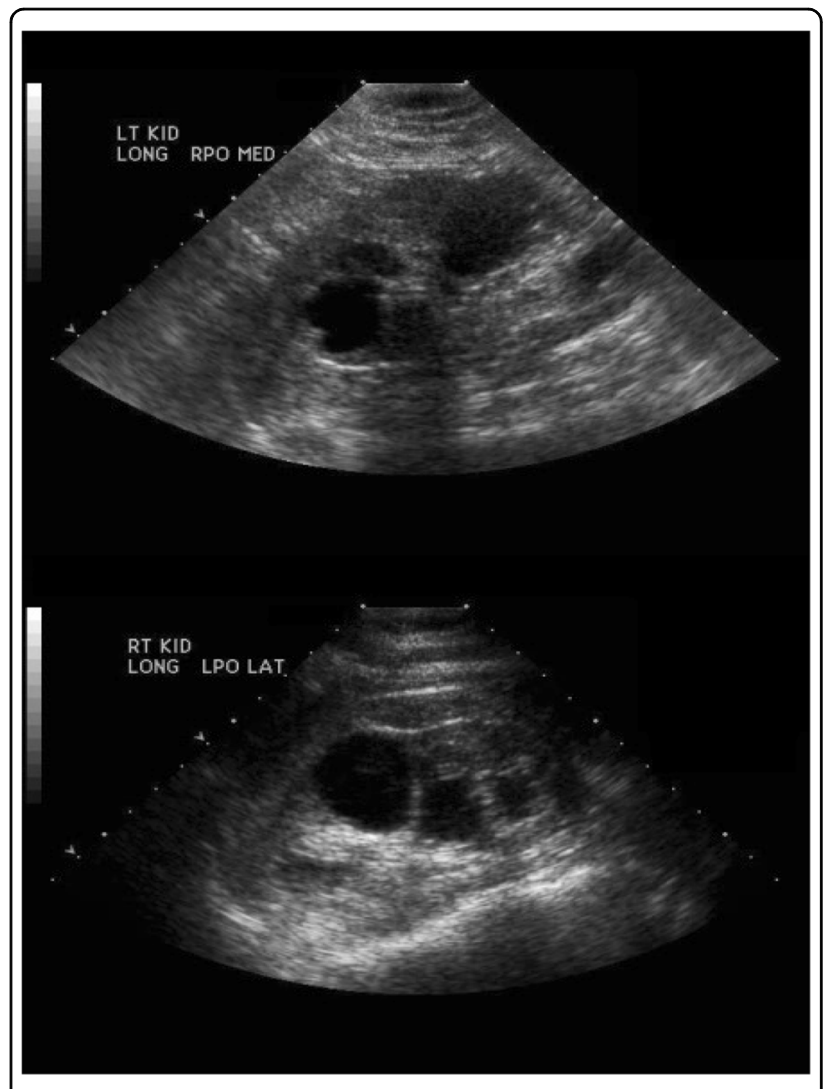

Fig. 1 Renal ultrasound showing multiple bilateral cysts

a compound heterozygote for ABCA4 variants. She has a heterozygous, paternally inherited, pathogenic variant in $A B C A 4$ (c.2588G>C, p.G863A) and a heterozygous, maternally inherited, likely pathogenic variant in $A B C A 4$ (c.203 C > G, p.P68R). These variants likely are the cause for the patient's macular degeneration and vision loss.

\section{Family 2}

Our second proband is a 48-year-old female with stage IV chronic kidney disease (estimated GFR of $22.5 \mathrm{~mL} /$ $\mathrm{min}$ ), nephrotic syndrome (6 $\mathrm{g}$ of protein/24-h urine) and hypertension. She underwent a renal biopsy, which revealed FSGS with patchy moderate tubular atrophy, interstitial fibrosis and mild chronic inflammation. She was initially started on prednisone but did not tolerate it due to excessive weight gain of up to 60 pounds and dyspnea. Her renal condition was treated with a combination of ACE inhibitor and ARB medications and excellent blood pressure control, which decreased her proteinuria to $250 \mathrm{mg} / 24-\mathrm{h}$ urine. Subsequently, her proteinuria started increasing again, and she was started on tacrolimus but showed poor response. She has a history of endometrial adenocarcinoma and endometrioid ovarian carcinoma. The patient also has a history of 


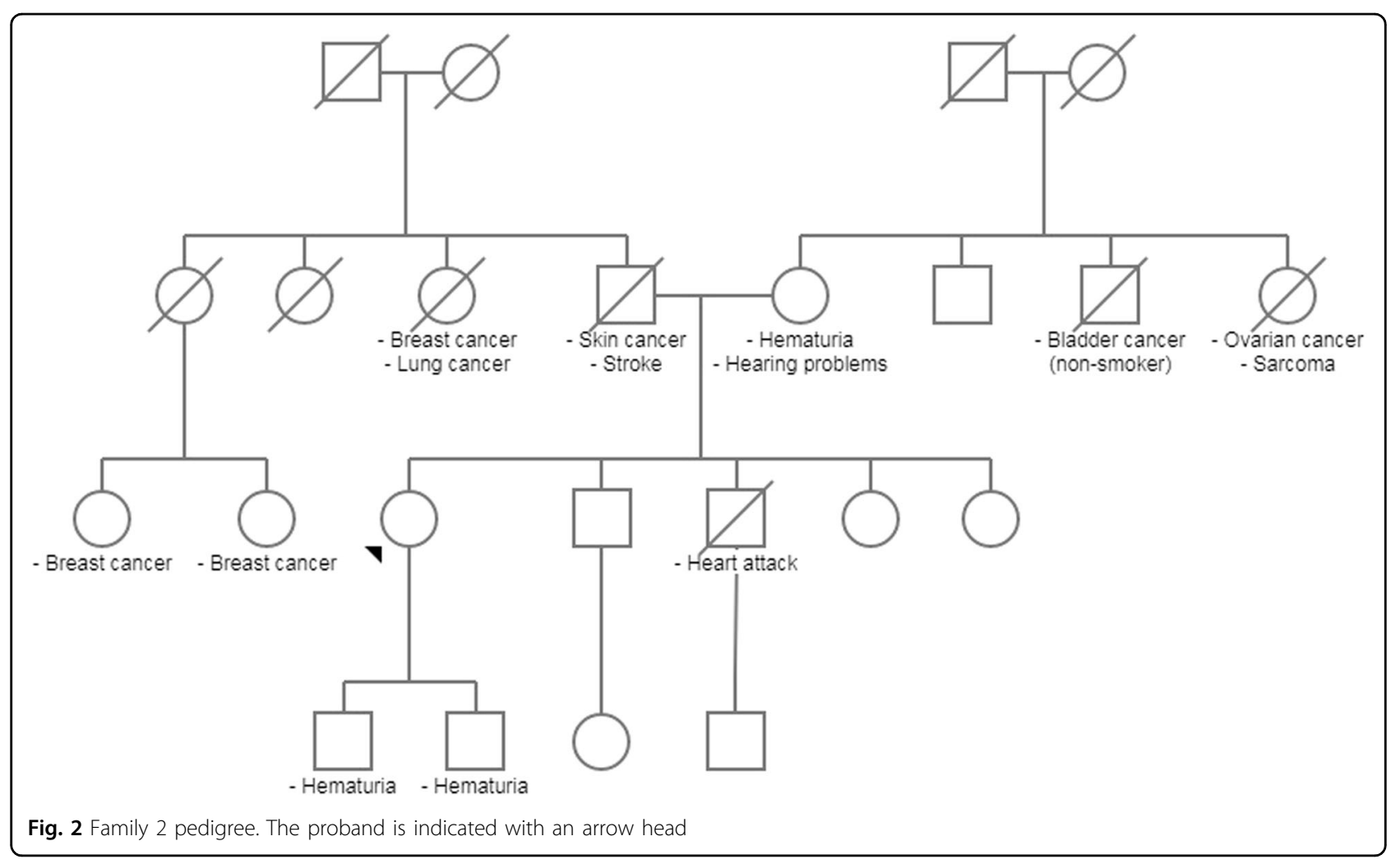

hypertension and obesity, as well as a family history of cancer (Fig. 2). The proband's mother and two sons have hematuria. The patient's sister carried a diagnosis of FSGS and stage IV chronic kidney disease (estimated GFR of $24 \mathrm{~mL} / \mathrm{min}$ ); her brother passed away at age 49 due to cardiac disease and was noted to have had hearing loss at a young age. Our proband was tested with a nephrotic syndrome/focal segmental glomerulosclerosis sequencing panel (Table 1). She was found to have a heterozygous, likely pathogenic variant in COL4A4 (c.2842G>T).

\section{Discussion}

Type IV collagen-related nephropathy, in which there is disruption of the normal glomerular basement membrane architecture and kidney disease, has been linked to pathogenic variants in COL4A3, COL4A4, and COL $4 A 5^{9}$. Heterozygous mutations in COL4A3 and COL4A4 result in mild disease with or without isolated microscopic hematuria $(\mathrm{MH})$ characterized by focal or diffuse thinning of the GBM, which is called thin basement membrane nephropathy (TBMN) ${ }^{9}$. Males hemizygous for COL4A5 pathogenic variants, and individuals of either sex with homozygous or compound heterozygous COL4A3/4 pathogenic variants, are at a greater risk of developing $\mathrm{X}$-linked and autosomal recessive Alport syndrome, respectively ${ }^{10}$. ESRD develops within the first three decades of life in AS, which is also associated with sensorineural deafness and ocular abnormalities, including asymptomatic dot and fleck retinopathy and lenticonus ${ }^{11,12}$. Females with heterozygous pathogenic variants in COL4A5 can present a variety of clinical features ranging from asymptomatic hematuria to more severe disease with progression to $\mathrm{ESRD}^{13}$. Moreover, pathogenic mutations in COL4A5 have been identified in families with familial and sporadic FSGS ${ }^{14}$. The presence of bilateral renal cysts in our patient is interesting, as, to the best of our knowledge, an association between COL4A5 variants and renal cysts has not been reported before.

In our first proband, WES detected a heterozygous variant in COL4A5 (c.141+1G>A, IVS2+1G>A). To the best of our knowledge, this variant has not been reported previously as either a pathogenic or a benign variant. This splice site variant destroys the canonical splice donor site in intron 2 and is predicted to cause abnormal gene splicing. The c.141+1G $>$ A variant has not been not observed in large population cohorts ${ }^{15-17}$. Based on the ACMG 2015 guidelines, the c c.141+1G>A, IVS2 $+1 \mathrm{G}>\mathrm{A}$ variant was classified as a pathogenic variant ${ }^{18}$. Even though our second proband had diffuse foot process effacement, her poor response to immunosuppression raised suspicion of a genetic etiology ${ }^{19}$. She was tested with a nephrotic syndrome/focal segmental glomerulosclerosis sequencing panel, which detected a 
Table 1 Nephrotic syndrome/focal segmental glomerulosclerosis sequencing panel

\begin{tabular}{|c|c|}
\hline Gene & OMIM ID \\
\hline ACTN4 & 604638 \\
\hline ANLN & 616027 \\
\hline ARHGAP24 & 610586 \\
\hline ARHGDIA & 601925 \\
\hline CD2AP & 604241 \\
\hline COL4A3 & 120070 \\
\hline COL4A4 & 120131 \\
\hline COL4A5 & 303630 \\
\hline COL4A6 & 303631 \\
\hline COQ2 & 609825 \\
\hline COQ6 & 614647 \\
\hline COQ8B & 615567 \\
\hline CRB2 & 609720 \\
\hline CUBN & 602997 \\
\hline DGKE & 601440 \\
\hline EMP2 & 602334 \\
\hline FAT1 & 600976 \\
\hline INF2 & 610982 \\
\hline ITGA3 & 605025 \\
\hline ITGB4 & 147557 \\
\hline KANK1 & 607704 \\
\hline KANK2 & 614610 \\
\hline KANK4 & 614612 \\
\hline LAMB2 & 150325 \\
\hline LMX1B & 602575 \\
\hline MYO1E & 601479 \\
\hline NEIL1 & 608844 \\
\hline NEXMIF & 300524 \\
\hline NPHS1 & 602716 \\
\hline NPHS2 & 604766 \\
\hline NUP107 & 607617 \\
\hline NUP205 & 614352 \\
\hline NUP93 & 614351 \\
\hline PAX2 & 167409 \\
\hline PDSS2 & 610564 \\
\hline PLCE1 & 608414 \\
\hline PTPRO & 600579 \\
\hline SCARB2 & 602257 \\
\hline SMARCAL1 & 606622 \\
\hline
\end{tabular}

Table 1 continued

\begin{tabular}{ll}
\hline Gene & OMIM ID \\
\hline TRPC6 & 603652 \\
TTC21B & 612014 \\
WDR73 & 616144 \\
WT1 & 607102 \\
XPO5 & 607845 \\
\hline
\end{tabular}

heterozygous and likely pathogenic variant in COL4A4 (c.2842G $>\mathrm{T})$. This variant is predicted to result in a premature protein termination (p.Gly948). This variant alone is probably sufficient to be the primary cause of the renal disease in this patient. Notably, heterozygous missense variants in COL4A4 and COL4A3 have been reported to cause familial or sporadic FSGS ${ }^{18,20}$.

While the first patient underwent WES, which detected a pathogenic variant in COL4A5, the second patient had a nephrotic syndrome/focal segmental glomerulosclerosis sequencing panel that revealed a likely pathogenic variant in COL4A4. There is an ongoing debate between the advantages and drawbacks of each test, especially in Mendelian disorders. One advantage of WES is variant segregation analysis and the concurrent testing of other family members, which can identify the pattern of Mendelian inheritance and detect other incidental variants that can be of clinical significance. Our first proband, thanks to WES, was found to be compound heterozygous for two ABCA4 variants. This finding helps in explaining her macular degeneration and vision loss and would be of great value when counseling this patient and her family. Gene panels have historically been preferred, especially when looking for genes related to a patient's phenotype, because of their low cost and short turnaround time, and many clinical geneticists consider them a rapid first-tier test.

Molecular genetic testing is noninvasive, can be highly accurate and is becoming the diagnostic procedure of choice for Alport syndrome since renal pathology findings of FSGS do not necessarily indicate genetic mutation ${ }^{21}$. Therefore, a multidisciplinary team approach, with expertize in clinical genetics, nephrology, and nephropathology, is important to attain an accurate diagnosis. While the rate of progression of renal disease may be related to the underlying causal mutation, molecular analysis may eventually provide more prognostic data than either renal or skin biopsy. Massively parallel (next generation) sequencing allows simultaneous analysis of the COL4A3, COL4A4, and COL4A5 genes, providing benefits in screening time and cost, and should be considered in similar cases to ours ${ }^{22,23}$. In summary, we present two families with novel pathogenic variants in COL4A5 and COL4A4 diagnosed by exome sequencing 
and gene panel testing, respectively, and discuss the merits of these approaches.

\section{Author details}

${ }^{1}$ Department of Internal Medicine, Mayo Clinic, Jacksonville, FL 32224, USA.

${ }^{2}$ Department of Clinical Genomics, Mayo Clinic, Jacksonville, FL 32224, USA

${ }^{3}$ Department of Nephrology, Mayo Clinic, Jacksonville, FL 32224, USA

\section{Conflict of interest}

The authors declare that they have no conflict of interest.

\section{Publisher's note}

Springer Nature remains neutral with regard to jurisdictional claims in published maps and institutional affiliations.

Received: 30 April 2018 Revised: 16 May 2018 Accepted: 17 May 2018. Published online: 10 July 2018

\section{References}

1. Mele, C. et al. MYO1E mutations and childhood familial focal segmental glomerulosclerosis. New Engl. J. Med. 365, 295-306 (2011).

2. D'Agati, V. D., Kaskel, F. J. \& Falk, R. J. Focal segmental glomerulosclerosis. New Engl. J. Med. 365, 2398-2411 (2011).

3. Kitiyakara, C., Kopp, J. B. \& Eggers, P. Trends in the epidemiology of focal segmental glomerulosclerosis. Semin. Nephrol. 23, 172-182 (2003).

4. Buscher, A. K. et al. Mutations in podocyte genes are a rare cause of primany FSGS associated with ESRD in adult patients. Clin. Nephrol. 78, 47-53 (2012).

5. Kashtan, C. E. Alport syndrome. An inherited disorder of renal, ocular, and cochlear basement membranes. Medicine 78, 338-360 (1999).

6. Boye, E. et al. Determination of the genomic structure of the COL4A4 gene and of novel mutations causing autosomal recessive Alport syndrome. Am. J. Hum. Genet. 63, 1329-1340 (1998).

7. Deltas, C., Savva, I., Voskarides, K., Papazachariou, L. \& Pierides, A. Carriers of autosomal recessive alport syndrome with thin basement membrane nephropathy presenting as focal segmental glomerulosclerosis in later life. Nephron 130, 271-280 (2015).

8. Malone, A. F. et al. Rare hereditary COL4A3/COL4A4 variants may be mistaken for familial focal segmental glomerulosclerosis. Kidney Int. 86, 1253-1259 (2014).
9. Torra, R., Tazón-Vega, B., Ars, E. \& Ballarín, J. Collagen type IV (a3-a4) nephropathy: from isolated haematuria to renal failure. Nephrol. Dial. Transplant. 19, 2429-2432 (2004).

10. Hudson, B. G., Tryggvason, K., Sundaramoorthy, M. \& Neilson, E. G. Alport's syndrome, Goodpasture's syndrome, and type IV collagen. New Engl. J. Med. 348, 2543-2556 (2003).

11. Lin, F. et al. Whole exome sequencing reveals novel COL4A3 and COL4A4 mutations and resolves diagnosis in Chinese families with kidney disease. BMC Nephrol. 15, 175 (2014).

12. $\mathrm{Wu}, \mathrm{Y}$. et al. A novel heterozygous COL4A4 missense mutation in a Chinese family with focal segmental glomerulosclerosis. J. Cell. Mol. Med. 20, 2328-2332 (2016)

13. Jais, J. P. et al. X-linked Alport syndrome: natural history and genotypephenotype correlations in girls and women belonging to 195 families: a "European Community Alport Syndrome Concerted Action" study. J. Am. Soc Nephrol. 14, 2603-2610 (2003).

14. Gast, $\mathrm{C}$. et al. Collagen $(\mathrm{CO} L 4 \mathrm{~A})$ mutations are the most frequent mutations underlying adult focal segmental glomerulosclerosis. Nephrol. Dial. Transplant. 31, 961-970 (2016)

15. Lek, M. et al. Analysis of protein-coding genetic variation in 60,706 humans. Nature 536, 285-291 (2016).

16. The Genomes Project C. A global reference for human genetic variation. Nature 526, 68 (2015)

17. Exome Variant Server NGESPE, Seattle, WA. http://evs.gs.washington.edu/EVS/.

18. Richards, S. et al. Standards and guidelines for the interpretation of sequence variants: a joint consensus recommendation of the American College of Medical Genetics and Genomics and the Association for Molecular Pathology. Genet. Med. 17, 405-424 (2015)

19. De Vriese, A. S., Sethi, S., Nath, K. A., Glassock, R. J. \& Fervenza, F. C. Differentiating primary, genetic, and secondary FSGS in adults: a clinicopathologic approach. J. Am. Soc. Nephrol. 29, 759-774 (2018).

20. Xie, J. et al. COL4A3 mutations cause focal segmental glomerulosclerosis 1. Mol. Cell Biol. 6, 498-505 (2014).

21. Inoue, $Y$. et al. Detection of mutations in the COL4A5 gene in over $90 \%$ of male patients with $x$-linked Alport's syndrome by RT-PCR and direct sequencing. Am. J. Kidney Dis. 34, 854-862 (1999).

22. Fallerini, $\mathrm{C}$. et al. Unbiased next generation sequencing analysis confirms the existence of autosomal dominant Alport syndrome in a relevant fraction of cases. Clin. Genet. 86, 252-257 (2014).

23. Moriniere, $\mathrm{V}$. et al. Improving mutation screening in familial hematuric nephropathies through next generation sequencing. J. Am. Soc. Nephrol. 25 2740-2751 (2014) 